\title{
Generating Power Using 3-Dimensional Graphene Foam
}

\author{
Wenbin Huang ${ }^{\text {a }}$, Wanling Li, Xiangjun Song, Yaozhou Liu, Peng Chen and \\ Xiaopan Liu
}

Center of Nanoscience and Technology, Institute of Mechanical Technology, Shijiazhuang, Hebei, 050003, China

aemail: huangwenbinde-2008@163.com

Keywords: generating power, 3-dimensional graphene, charge carrier, polarity

\begin{abstract}
In this work, the electrical conductance and induced current of 3-dimensional graphene foam (GF) are investigated when the mixture of water and ethanol flows through it. When different mixing ratios of ethanol: water (ethanol: water $=25: 75,50: 50,75: 25$, and 100:0 by volume) flow through the GFs, their electrical conductance are almost the same as that of original GF. Meanwhile, the induced current can be obtained when the mixture flows through the GF. The direction of induced current depends on that of the flow of mixture, the value of induced current has no matter with the flow direction of mixture but is closely related to the flow velocity and polarity of mixture. The mechanism of the induced electricity is discussed, which is attributed to the coupling of flowing solution molecules with the charge carriers of graphene at the solid/liquid interface. These results indicate that GFs have a bright potential application in realizing the self-powered function of nano/micro electromechanical systems (N/MEMS) in many special environments.
\end{abstract}

\section{Introduction}

Harvesting energy from the ambient environment directly is one of the most promising and effective approaches for powering nano/micro electromechanical systems (N/MEMS), including ultra-sensitive chemical sensors, implantable biosensors, portable/wearable personal electronics, and even remote and mobile environmental sensors. Carbon nanomaterials, which are responsible for the conversion of some ambient energy into electrical energy directly, have attracted much attention recently. It has been demonstrated that the flow of polar liquids over carbon nanotubes (CNTs) can generate a net potential difference and associated electric current in the nanotubes along the flow direction. The induced voltage increases with flow velocity to a saturated value that depends on the ionic conductivity and the polar nature of liquids.

Graphene, a two-dimensional crystal with carbon atoms arranged in a hexagonal structure, has attracted immense interests since it was experimentally discovered. [1] Whether energy can be also harvested when liquids flow over graphene? What may affect the output power? Recently, different research groups have performed related researches. In 2011, it was reported that a small piece of graphene sheet about $30 \times 16 \mu \mathrm{m}^{2}$ immersed in flowing water with $0.6 \mathrm{M}$ hydrochloric acid can produce voltage $\sim 20 \mathrm{mV}$. In 2013, Lee et al. reported that voltage generation could be induced in non-ionic liquids over monolayer graphene. Recently, we have reported that electricity can be induced when pure water flows through 3-dimensional graphene foam (GF). [2] As 3-dimensional structure, GFs can make full use of specific surface area. Besides, the high quality of the graphene sheets and their perfect connection in GFs give the material excellent characters, e. g., outstanding electrical and mechanical properties, etc.

In this paper, we designed a new experiment, in which water is replaced by the mixtures of water and ethanol with different volume ratios, which are ethanol: water $=25: 75$, 50:50, 75:25 and 100:0. The electrical conductance and induced current of GFs were investigated when the mixtures flowed through them. For different mixing ratios, the current-voltage (I-V) curves were almost parallel to each other, indicating that the electrical conductivities of GFs were the same. Take the volume mixing ratio of ethanol: water $=25: 75$ as example, when the mixture flowed through GF, electricity 
can be induced. The direction of induced current depends on that of the flow of mixture, the value of induced current has no matter with the flow direction of mixture but is closely related to the flow velocity and polarity of mixture. The mechanism was studied and attributed to the coupling of solution molecules with the carriers of graphene at the solid/liquid interface.

\section{Experiment}

GFs were synthesized on a scaffold of porous nickel $(5 \mathrm{~cm} \times 5 \mathrm{~cm} \times 1 \mathrm{~cm})$ by chemical vapor deposition (CVD).[3] Firstly, we rolled up the porous nickel and put it on a half round quartz tube, then inserted it into the horizontal tube furnace ( $32 \mathrm{~mm}$ inner diameter), which was used as sample growth chamber). At $\sim 1000{ }^{\circ} \mathrm{C}$ under ambient pressure, carbon atoms were deposited on the nickel foam through $\mathrm{CH}_{4}$ decomposition. After growing for about 10 minutes, a continuous GF formed as a macroscopic structure, and there were extremely thin graphene sheets outside the porous nickel. The graphene sheets spread on the entire surface of porous nickel and there were no physical breaks in the network. In order to make sure that the scaffold of porous nickel could be put into the pipe exactly and most of the solution molecules flow through the pores, it was coiled closely into a roll $(\Phi 15 \times 50 \mathrm{~mm})$ along the sidelines.18

Raman spectroscopy is one of the powerful and convenient tools to characterize carbon nanomaterial. [4] By using the laser excitation of $514 \mathrm{~nm}$ of an argon ion laser, the Raman spectra of our samples were collected. To avoid damage on the samples, the laser power was set to be less than $1 \mathrm{~mW}$. A $\sim 1 \mu \mathrm{m}$ laser spot size can be obtained with the application of a $100 \times$ objective lens with a numerical aperture of 0.90 . From the intensity ratio of $G$ and 2D modes of the as-grown GF, it can be concluded that the walls of GF are comprised of monolayer to few-layer graphene sheets.

In order to measure the I-V characteristics and induced currents of GFs, we designed a test facility, which was schematically shown in Figure 1(a). A long hollow tube was connected to a flow control valve and an overhead tank. By adjusting the height of the flow control valve and the overhead tank, the flow velocity of solution was varied. We attached two metal wires with insulating skin to both ends of the GF roll, and then the GF roll was inserted into the test tube. Through controlling the flow velocity and changing the mixing ratios of solution, which are water: ethanol $=25: 75,50: 50,75: 25$ and 100:0 by volume, the electrical conductance and induced currents of GFs were measured by a semiconductor characterization system called Keithley 4200-SCS (voltage resolution $\sim 1 \mu \mathrm{V}$ ).
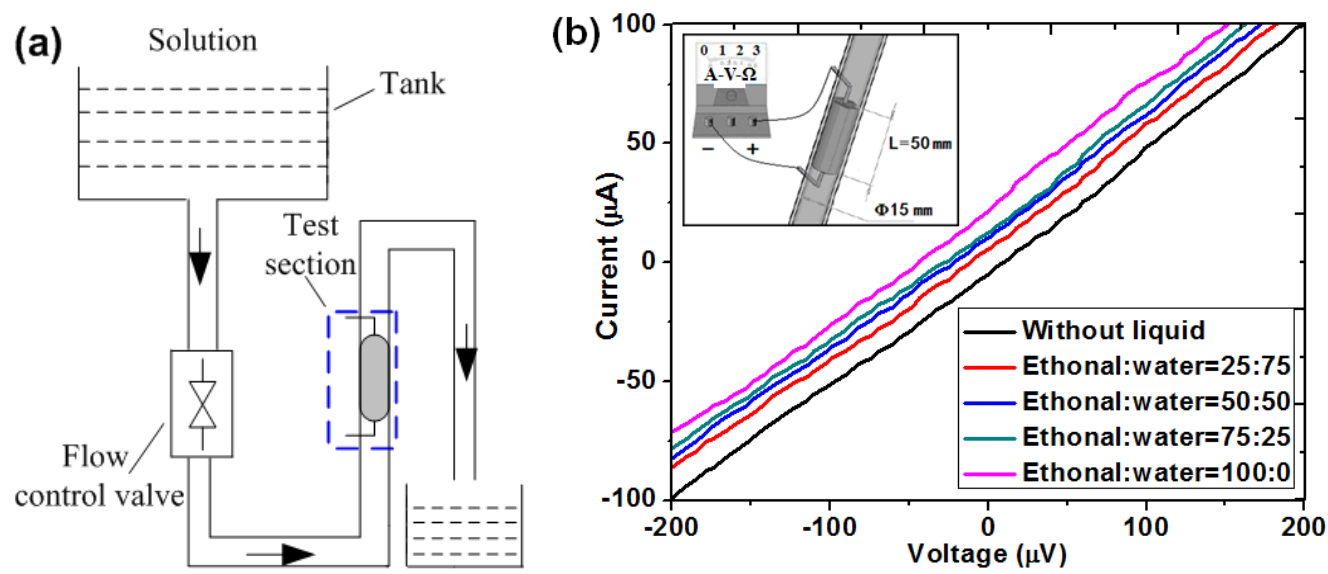

Fig. 1. (a) The schematic layout of the flow test facility. (b) The current-voltage characteristics of GFs when the mixtures with different ratios (ethanol: water $=25: 75,50: 50,75: 25$ and 100:0 by volume) flow through.

\section{Results and Discussion}

The electrical conductivity and induced currents of GFs have been investigated when the mixtures flow through. As shown in Figure 1(b), for a given mixing ratio, the I-V curve can be well fitted using a linear function. Meanwhile, for different mixing ratios, all the I-V curves are almost parallel to each 
other, indicating that the electrical conductivity has no change. Furthermore, we can calculate the electrical conductance correspondingly by $\delta=\Delta \mathrm{I} / \Delta \mathrm{V}$ (I and $\mathrm{V}$ are the induced current and voltage, respectively), which is $\sim 0.5 \mathrm{~s}$.
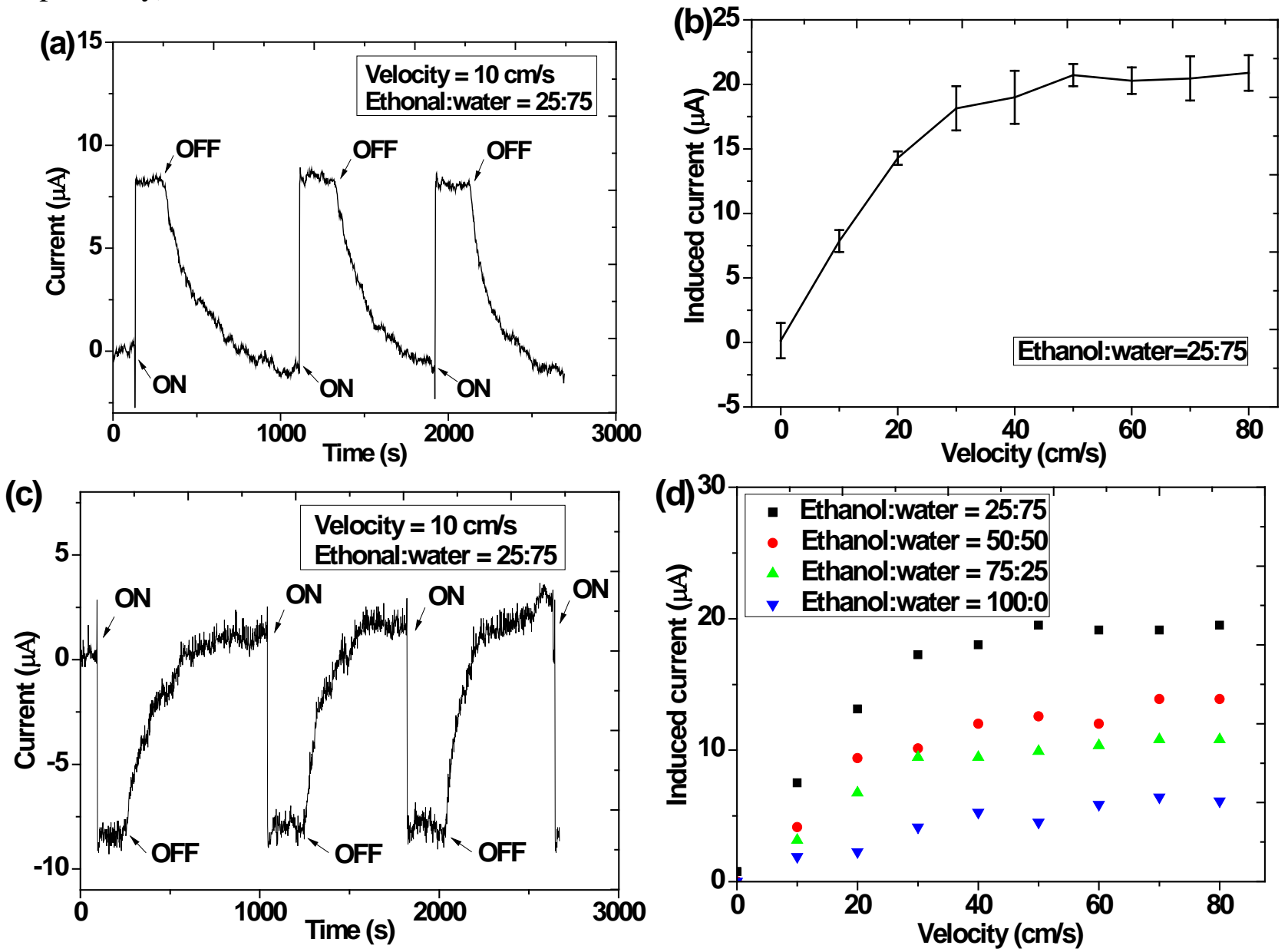

Fig. 2. (a) The output current when the flow velocity of the mixture (ethanol: water $=25: 75$ by volume) is $10 \mathrm{~cm} / \mathrm{s}$ and bias voltage is $0 \mu \mathrm{V}$. (b) The variation of induced current in the range of velocity of 0 to $80 \mathrm{~cm} / \mathrm{s}$. (c) The output current when the flow velocity and bias voltage are identical with (a) but the flow direction is opposite. (d) (b) The variation of induced currents as the flow velocity changes in the range of 0 to $80 \mathrm{~cm} / \mathrm{s}$ when the bias voltage is $0 \mu \mathrm{V}$ and the mixing ratios of solution are ethanol: water $=25: 75,50: 50,75: 25$, and 100:0 by volume, respectively.

We have taken the volume mixing ratio of ethanol: water $=25: 75$ as example, and carried out the electrical measurements of our sample in the mode of voltage bias. As shown in Figure 2(a), a current of $\sim 8 \mu \mathrm{A}$ was induced when the bias voltage was set as $0 \mu \mathrm{V}$ and the flow velocity of solution was 10 $\mathrm{cm} / \mathrm{s}$, the character was very repeatable. It was found that the induced current was closely related to the flow velocity of solution, which can be inferred from Figure 2(b). When the mixture of water and ethanol flowed in the GFs, the polar molecules of solution would couple with free charge carriers of graphene at the interface. Because of the friction at the solid/liquid interface, continuous coupling and separation happened between the polar molecules and free charge carriers, which led to a nonlinear relationship between the drift velocity of free charge carriers and the bulk flow velocity. The linear velocity of solution (v) can be calculated by the following equation:

$v=V_{l} / \Delta t \cdot S$

Where $\mathrm{V}_{\mathrm{l}}$ is the volume of solution flowing into the can during the time interval $\Delta \mathrm{t}, \mathrm{S}$ is the flow cross sectional area of solution in the pipe, which can be calculated by $\pi r^{2}$ ( $r$ is the inner radius of the pipe). The induced current is nearly $0 \mu \mathrm{A}$ when the flow velocity of solution is $0 \mathrm{~cm} / \mathrm{s}$. In the range of flow velocity of 0 to $60 \mathrm{~cm} / \mathrm{s}$, the induced current becomes larger with the increase of flow velocity, and shows dependent on the flow velocity of solution nonlinearly. For example, when the flow velocity is $10 \mathrm{~cm} / \mathrm{s}$, the induced current is $\sim 8 \mu \mathrm{A}$. The induced current becomes $\sim 14 \mu \mathrm{A}$ when the flow velocity is set as $20 \mathrm{~cm} / \mathrm{s}$. However, once the flow velocity exceeds $60 \mathrm{~cm} / \mathrm{s}$, the induced current tends to a 
saturated value of $\sim 20 \mu \mathrm{A}$. In order to make sure the effect of the direction of induced current, a control experiment has been done, in which the flow direction of solution was reversed. In Figure 2(c), it is obvious that the induced current goes in the opposite direction, indicating that the direction of induced current is dominated by the flow direction of solution. It means that the induced current is positive when the solution flows from positive terminal to GND. In contrast, when the solution flows from GND to positive terminal, the induced current becomes negative. Meanwhile, the induced current measured is very repeatable.

To further explore the relationship between the bias voltage and the induced current, the induced currents have been measured at different bias voltages. The induced current was $\sim 8 \mu \mathrm{A}$ when the bias voltage was defined at $10 \mu \mathrm{V}$ and the flow velocity of solution was $10 \mathrm{~cm} / \mathrm{s}$. From Figure 2(d), it can be known that the induced current has no obvious change when the flow velocity of solution was defined at a certain value despite of the bias voltage varying in the negative and positive range. Hence the induced current is independent of the external bias voltage.

\section{Summary}

In conclusion, the electrical conductance and induced currents of GFs were investigated when the mixture of water and ethanol flowed through them. The electrical conductivity of GF had no change as the mixing ratios of ethanol and water differed. When the mixture flowed through GF, electricity can be induced. The flow of mixture determined the direction of induced current, but had no effect on the value of induced current. The induced current was found nonlinearly dependent on the flow velocity of solution in the range of 0 to $60 \mathrm{~cm} / \mathrm{s}$. When the flow velocity exceeded $60 \mathrm{~cm} / \mathrm{s}$, the induced current tended to a saturated value. The polarity of solution was found to have positive correlation with the induced current. The mechanism was studied and attributed to the coupling of flowing solution molecules with the charge carriers of graphene at the solid/liquid interface. The experimental results indicated that GFs have a bright application prospect in realizing the self-powered function of N/MEMS. Besides, the unique network structure of GF can enable many other applications, including elastic and flexible conductors, high-performance electrically conductive polymer composites, electrode materials for lithium ion batteries and supercapacitors, etc.

\section{Acknowledgements}

The authors sincerely thank National Center for Nanoscience and Technology for providing the fine work environment, experimental facility and technical support. This work was supported by National Science Foundation of China (Grant Nos. 10774032, 90921001).

\section{References}

[1] F Yu, L.J. Hu, H.Q. Zhou, C.Y. Qiu, H.C. Yang, M.J. Chen and L.F. Sun: J. Nanosci. Nanotech. Vol. 1335-1338 (2013), p. 13.

[2] Q. Yuan and Y.P. Zhao: J. Am. Chem. Soc., Vol. 6374-6376 (2009), p. 131.

[3] Z. Liu, K.H. Zheng, L.J. Hu, J. Liu, C.Y. Qiu, H.Q. Zhou, H. Huang, H.C. Yang, M. Li, C. Gu and L.F. Sun: Adv. Mater. Vol. 999-1003 (2010), p. 22.

[4] F. Yavari, Z. Chen, A.V. Thomas, and W. Ren: Sci. Rep. Vol. 1-15 (2011), p. 1.

[5] Y.P. Zhao, H.Q. Zhou, K.H. Zheng, and W. Ren: Adv. Mater. Vol. 171 (2012), p. 175-178. 